\title{
Isospin symmetry breaking effects in the pion and nucleon masses
}

\author{
Ran Zhou*t \\ Physics Department, University of Connecticut, Storrs, CT 06269-3046, USA \\ E-mail: zhouran@phys.uconn.edu
}

\section{Thomas Blum}

Physics Department, University of Connecticut, Storrs, CT 06269-3046, USA

RIKEN BNL Research Center, Brookhaven National Laboratory, Upton, New York 11973, USA

E-mail: tblumephys.uconn.edu

\section{Takumi Doi}

Department of Physics and Astronomy, University of Kentucky, Lexington, KY 40506, USA

E-mail: doieribf.riken.jp

\section{Masashi Hayakawa}

Department of Physics, Nagoya University, Nagoya 464-8602, Japan

E-mail: hayakawa@eken.phys.nagoya-u.ac.jp

\section{Taku Izubuchi}

Institute for Theoretical Physics, Kanazawa University, Kanazawa 920-1192, Japan

RIKEN BNL Research Center, Brookhaven National Laboratory, Upton, New York 11973, USA

E-mail: izubuchi@quark.phy.bnl.gov

\section{Norikazu Yamada}

High Energy Accelerator Research Organization(KEK), Tsukuba, Ibaraki 305-0801, Japan

The Graduate University for Advanced Studies, Tsukuba, Ibaraki 305-0801, Japan

E-mail: norikazu.yamada@kek.jp

We investigate the electromagnetic mass splittings in the pseudoscalar meson and nucleon systems by combining $2+1$ flavor domain wall fermion gauge configurations, generated by the RBC and UKQCD collaborations, and quenched, non-compact, lattice QED configurations. We analyze finite volume effects by using $16^{3} \times 32$ and $24^{3} \times 64$ lattices.

The XXVI International Symposium on Lattice Field Theory

July 14-192008

Williamsburg, Virginia, USA

\footnotetext{
* Speaker.

${ }^{\dagger}$ We thank Riken, Brookhaven National Laboratory and the U.S. Department of Energy for providing the facilities and hospitality where this work was done.
} 


\section{Introduction}

The mass splitting in the meson and baryon system is an interesting topic in hadron spectroscopy. It is related with the quark masses which are the fundamental parameters of the Standard Model. The mass difference of the proton and neutron is also crucial to the phenomenological model of nuclei, because it plays an important role in the neutron $\beta$ decay process. These mass differences have already been measured by experimentalists to good accuracy. So they can be used as input to help theorists to determine the parameters in their phenomenological models or check the validity of the theory.

The mass of the hadron is determined by both QED and QCD dynamics. For the QCD interaction, since the coupling constant is too large at the low energy regime, the perturbation theory is not applicable. People have developed all kinds of effective theories of QCD from the 1950s. They can be used to describe the hadron mass spectrum. For instance, the mass of the pseudoscalar meson, especially for the pion, is much smaller than the mass of the baryon. It can be explained by the spontaneous breakdown of chiral symmetry. When QED is included, the meson masses are split, but $m_{\pi^{+}}^{2}-m_{\pi^{0}}^{2} \simeq m_{K^{+}}^{2}-m_{K^{0}}^{2}$, which is explained by Dashen's theorem [1]. Partially quenched chiral perturbation theory $(\mathrm{PQ} \chi \mathrm{PT})$ gives a more general description of $\mathrm{QCD}$ phenomena: valence and sea quark masses are varied independently. It is important since the cost to generate the dynamical QCD configurations for a large lattice is still pretty high today.

Recent developments in PQ $\chi$ PT [2] allows us to incorporate the QED interaction too, so we can investigate the mass splitting which is related to the QED interaction. Since the QED interaction is much weaker than the QCD interaction, it will only provide a small shift in the hadron mass from its QCD value. But this shift is important to the splitting in the pion and kaon multiplet.

Because the lattice calculation can give the hadron mass spectrum from first principles, it is used to test whether the QCD+QED interaction can actually reproduce the hadron spectrum. The pioneering work on this topic has been done in [3] with the quenched QCD and non-compact QED configuration. The development of Lattice QCD enables us to investigate the hadron spectrum with 2+1 flavor dynamical QCD configurations, which means we can include the effect of the degenerate up and down quarks and also the heavier strange quark in the sea quark sector. So our simulation result can be used to fit the PQ $\chi \mathrm{PT}$ formula. We can extract the unknown low energy constants(LEC's) from the fitting. After we know the LEC's, we can use the pseudoscalar meson mass as input to determine the physical value of the $u, d$, and $s$ quark masses. The MILC group also reports their analysis of the mass splitting using staggered fermion ensembles in Basak's Talk.

The lattice simulation is also used for the mass splitting in the nucleon system [4]. We present preliminary results for the mass difference between the proton and neutron due to QED.

\section{Theoretical Background}

In chiral perturbation theory of QCD, the mass square of the pseudoscalar meson is determined by the spontaneous breakdown of chiral symmetry from $S U(3)_{L} \otimes S U(3)_{R} \otimes U(1)_{V}$ to $S U(3)_{V} \otimes$ $U(1)_{V}$. The Goldstone boson generated by this mechanism is the pseudoscalar meson octet. All of them are massless in the chiral limit. If the QED interaction is also considered, the symmetry group will be broken further to $S U(2)_{V} \otimes U(1)_{e m} \otimes U(1)_{V}$. The only Goldstone bosons left are the 
neutral pion and kaons (the neutral pion can be considered a Goldstone boson if $\mathscr{O}\left(\alpha^{2}\right)$ terms are neglected[8]).

Since we calculate with several valence quark mass and charge combinations, we use PQ $\chi \mathrm{PT}$. Suppose we have a "kaon" which is composed by $u$ and $s$ quarks. Following the notation of [2], index 1 denotes the $u$ quark and 3 the s quark. The square of the meson mass is[2]

$$
\begin{gathered}
m^{2}=\chi_{13}+\frac{2 C e^{2}}{F_{0}^{2}}\left(q_{1}-q_{3}\right)^{2}+\frac{\delta^{(4)}}{F_{0}^{2}} \\
\frac{\delta^{(4)}}{F_{0}^{2}}=\left[\left(48 L_{6}^{r}-24 L_{4}^{r}\right) \bar{\chi}_{1} \chi_{13}+\left(16 L_{8}^{r}-8 L_{5}^{r}\right) \chi_{13}^{2}-\frac{1}{3} \bar{A}\left(\chi_{m}\right) R_{n 13}^{m} \chi_{13}-\frac{1}{3} \bar{A}\left(\chi_{p}\right) R_{q \pi \eta}^{p} \chi_{13}\right] / F_{0}^{2} \\
+e^{2} \bar{A}\left(\chi_{13}\right) q_{13}^{2}+4 e^{2} \bar{B}\left(\chi_{\gamma}, \chi_{13}, \chi_{13}\right) q_{13}^{2} \chi_{13}-4 e^{2} \bar{B}_{1}\left(\chi_{\gamma}, \chi_{13}, \chi_{13}\right) q_{13}^{2} \chi_{13} \\
-C e^{2}\left[-48 L_{4}^{r} q_{13}^{2} \bar{\chi}_{1}-16 L_{5}^{r} q_{13}^{2} \chi_{13}+2 \bar{A}\left(\chi_{1 s}\right) q_{1 s} q_{13}-2 \bar{A}\left(\chi_{3 s}\right) q_{3 s} q_{13}\right] / F_{0}^{4} \\
-Y_{1} 4 e^{2} \bar{Q}_{2} \chi_{13}+Y_{2} 4 e^{2} q_{p}^{2} \chi_{p}+Y_{3} 4 e^{2} q_{13}^{2} \chi_{13}-Y_{4} 4 e^{2} q_{1} q_{3} \chi_{13}+Y_{5} 12 e^{2} q_{13}^{2} \bar{\chi}_{1}
\end{gathered}
$$

$Y_{i}$ are independent linear combinations of the LEC's $K^{E r}$ as defined in Eq. (48) of [2], and $\chi_{i}$ is the LO meson mass, $\chi_{i}=2 B_{0} m_{i}, \chi_{i j}=\left(\chi_{i}+\chi_{j}\right) / 2, q_{13}=q_{1}-q_{3}=q_{u}-q_{s}$. The form of the functions $\bar{A}, \bar{B}, \bar{B}_{1}, R$, and others can be found in [2].

\begin{tabular}{|c|c|c|}
\hline & QCD LEC's & QED LEC's \\
\hline LO & $B_{0}, F_{0}$ & $C$ \\
\hline NLO & $L_{4}^{r}, L_{5}^{r}, L_{6}^{r}, L_{8}^{r}$ & $C, Y_{1}, Y_{2}, Y_{3}, Y_{4}, Y_{5}$ \\
\hline
\end{tabular}

Table 1: LEC's in PQ $\chi$ PT

Table 1 shows all of the LEC's which are necessary to determine the pseudoscalar meson mass at NLO. The QCD LEC's have been computed in[7], so the current simulation is used to extract the QED LEC's. Note that the definition of the decay constant used in [2] (followed here) is $\sqrt{2}$ smaller than the one in [7]. For the splitting in baryon system, we focus on the mass differences between the proton and neutron. Here, we study only the degenerate case, $m_{u}=m_{d}$. Then the leading contribution to the mass difference is proportional to $\alpha$.

\section{Lattice Simulation}

Following[3], the lattice calculation employs combined QCD+QED gauge configurations. For the QCD configurations, we use the $N_{f}=2+1$ flavor QCD configurations generated with the domain wall fermion and Iwasaki gauge actions by the RBC and UKQCD collaborations [5, 6, 7]. For the $16^{3} \times 32$ lattice, we use light quark mass $0.01,0.02$ and 0.03 ensembles. For $24^{3} \times 64$ lattice, we use the $0.005,0.01,0.02$ and 0.03 ensembles. The strange quark mass is fixed to 0.04 in all cases. Table 2 gives the details of the lattice ensembles used in this work.

The QED configurations are generated in a quenched, non-compact form $[8,9]$. Here we employ the Feynman gauge instead of the Coulomb gauge which was used previously[8]. One advantage of the non-compact QED formalism is that the $U(1)$ gauge potential $A_{\mu}$ can be chosen 


\begin{tabular}{ccccccc}
\hline lat & $m_{\text {sea }}$ & $m_{\text {val }}$ & Trajectories & $\Delta$ & $N_{\text {meas }}$ & $t_{s r c}$ \\
\hline $16^{3}$ & 0.01 & $0.01-0.03$ & $500-4000$ & 20 & 176 & 0 \\
$16^{3}$ & 0.02 & $0.01-0.03$ & $500-4000$ & 20 & 176 & 0 \\
$16^{3}$ & 0.02 & $0.01-0.03$ & $500-4000$ & 20 & 176 & 0 \\
\hline $24^{3}$ & 0.005 & $0.005-0.03$ & $900-8060$ & 40 & 180 & 0 \\
$24^{3}$ & 0.01 & $0.01-0.03$ & $1460-5040$ & 20 & 180 & 0 \\
$24^{3}$ & 0.02 & 0.02 & $1800-3580$ & 20 & 180 & 0,32 \\
$24^{3}$ & 0.03 & 0.03 & $1800-3580$ & 20 & 180 & 0,32 \\
\hline
\end{tabular}

Table 2: QCD gauge configuration ensembles generated by the RBC and UKQCD collaborations [5, 6, 7]. $\Delta$ is the separation of the trajectory number in molecular dynamics time units. $\beta_{\mathrm{QCD}}=2.13$. The inverse lattice scale is $a^{-1}=1.729(28) \mathrm{GeV}$ for both of $16^{3}$ and $24^{3}$ lattice.

randomly with the correct distribution in momentum space, then Fourier transformed to coordinate space, so there are no autocorrelations in the ensemble. Another advantage is that there is no (lattice artifact) photon self-interaction in the action. To couple $A_{\mu}$ to the fermions, the non-compact field is exponentiated in the usual way, so the combined gauge field appears as an $S U(3)$ matrix times a phase, $U_{\mu}^{Q C D}(x) \times U_{\mu}^{Q E D}(x)$.

Since the QED interaction does not have confinement, it is possible that the finite volume can induce a significant systematic error. So, we do our simulation on both $16^{3}$ and $24^{3}$ lattice configurations with the same lattice spacing. Then we can investigate the finite volume effect in the mass spectrum.

\section{Numerical Results}

We can switch on and off the QED interaction by setting the charge $e \neq 0$ and $e=0$. We first extract the masses from wall source, point sink, correlation functions. We define the mass difference, $\delta m^{2}=m^{2}(e \neq 0)-m^{2}(e=0)$, and then fit $\delta m^{2}$ with the PQ $\chi \mathrm{PT}$ formula, using the standard jackknife method to estimate the statistical error. $\delta m^{2}$ is averaged over $+e$ and $-e$ ensembles to decrease the noise in the signal [8]. We take the QCD LEC's from the $S U$ (3) PQ $\chi$ PT fit in [7] since we use the same ensembles. We also use these for the $16^{3}$ case because these two ensembles have the same lattice scale. Then we fit our data to obtain all of the QED LEC's, except $Y_{1}$ because the sea quarks are not coupled to photons. Thus, $Y_{1}=0$ in this work.

$\mathrm{PQ} \chi \mathrm{PT}$ is based on chiral symmetry, which is not exact in the domain wall fermion formalism with finite $L_{s}$. To lowest order, the violation of chiral symmetry can be counted as a small shift in the input quark mass, so we substitute $m_{i}+m_{\text {res }}$ for all quark masses, where the residual mass $m_{\text {res }}$ is determined from the method in reference [8]. Also following [8], we add additional LEC's, $C_{1}$ and $C_{2}$, proportional to $\left(q_{1}-q_{3}\right)^{2}$ and $\left(q_{1}+q_{3}\right)^{2}$, respectively, to account for chiral symmetry breaking due to finite $L_{s}$ at order $\mathscr{O}(\alpha)$. These LEC's vanish when $L_{s} \rightarrow \infty$. $C_{1}$ mixes with the LEC $C$ in Eq. (2.2), so it must be subtracted to obtain the physical LEC. $C_{1}$ is measured from $m_{\text {res }}$ with $e \neq 0$ as described in [8]. For the preliminary results presented here, this subtraction has not been performed. 
By fitting the results for $\delta m^{2}$, we can get all of the QED LEC's, including the pure lattice artifact $C_{2}$. The fit results are shown in Figure 1 along with the unitary data points (though all of the partially quenched data was used in the fit) and tabulated in Table 3 . We perform fits with and without chiral logs. $\chi^{2} /$ dof is adequate in either case, except for $24^{3}$ when all the quark mass data is fitted. If the heaviest sea quark points are omitted, $\chi^{2}$ becomes reasonable. The main effect of the $\operatorname{logs}$ is to significantly reduce the value of the charged meson splitting in the chiral limit. Note, the $\log$ terms vanish for the neutral mesons, and the neutral splittings should also vanish in the chiral limit (see [8] for a detailed discussion). The non-vanishing chiral limit value in the plot is due of the $C_{2}$ term, or the explicit chiral symmetry breaking induced by finite $L_{s}$.
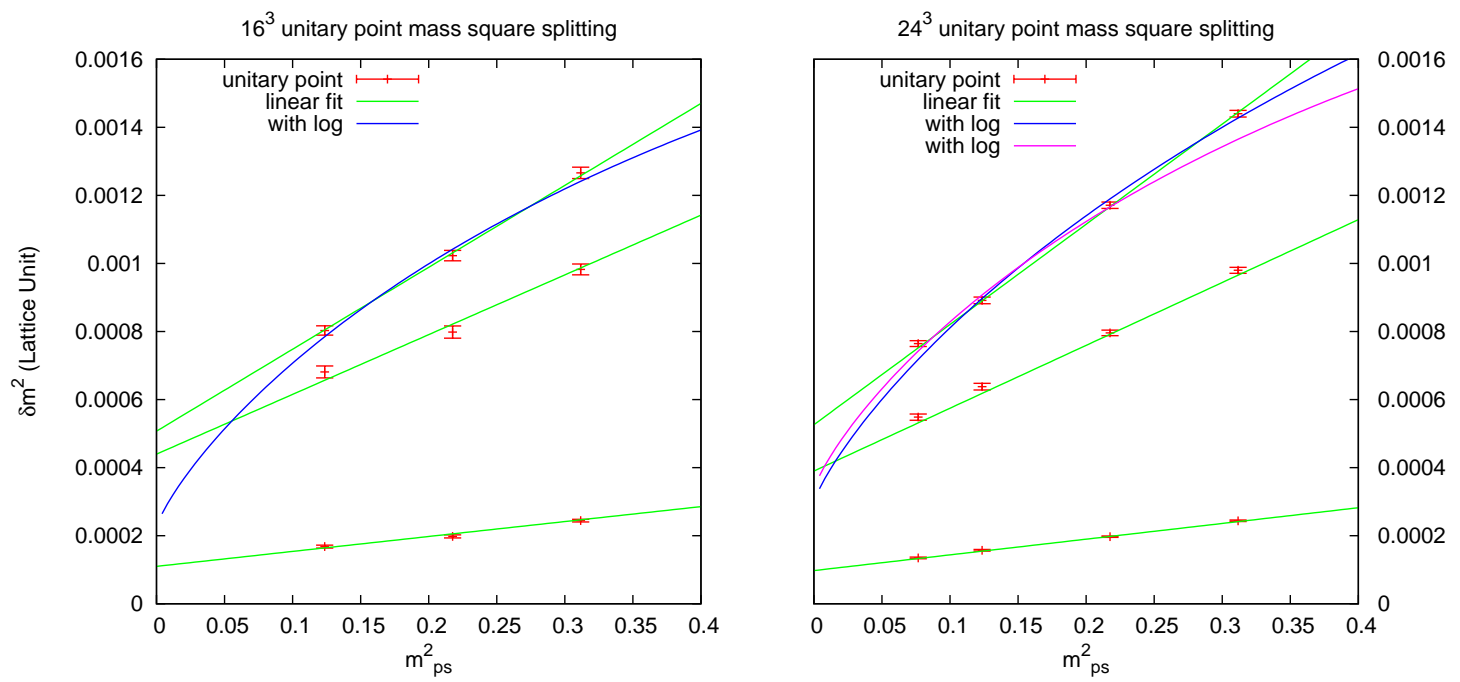

Figure 1: $16^{3}$ (left panel) and $24^{3}$ (right panel) lattice data points and fit results for the meson mass splitting. Lines correspond to fits with and without chiral logs. The data points in the plot correspond to $u \bar{d}, u \bar{u}$ and $d \bar{d}$ mesons, respectively, from top to bottom.

\begin{tabular}{|c|c|c|c|c|c|c|}
\hline & $16^{3}$ & $24^{3}$ & $16^{3}$ & $24^{3}$ & $24^{3}$ & Ref. [2] \\
\hline with log & No & No & Yes & Yes & Yes & Yes \\
\hline fit range & $0.01-0.03$ & $0.005-0.03$ & $0.01-0.03$ & $0.005-0.03$ & $0.005-0.02$ & N/A \\
\hline$C \times 10^{6}$ & $3.48(40)$ & $3.02(14)$ & $0.85(16)$ & $0.769(64)$ & $0.96(10)$ & 2.71 \\
\hline$Y_{2} \times 10^{2}$ & $1.47(3)$ & $1.51(1)$ & $1.56(3)$ & $1.54(1)$ & $1.51(2)$ & 0.53 \\
\hline$Y_{3} \times 10^{3}$ & $7.64(72)$ & $7.61(39)$ & $-1.08(45)$ & $-1.17(35)$ & $-2.79(43)$ & 2.65 \\
\hline$Y_{4} \times 10^{3}$ & $8.00(78)$ & $7.55(50)$ & $9.71(92)$ & $8.22(51)$ & $8.85(80)$ & 5.7 \\
\hline$Y_{5} \times 10^{3}$ & $1.48(23)$ & $2.43(12)$ & $1.05(24)$ & $2.23(12)$ & $2.35(19)$ & 0 \\
\hline$C_{2} \times 10^{3}$ & $2.69(10)$ & $2.39(5)$ & $2.69(10)$ & $2.39(5)$ & $2.48(6)$ & N/A \\
\hline$\chi^{2} /$ dof & $0.7(6)$ & $0.6(2)$ & $1.0(6)$ & $2.8(5)$ & $1.2(6)$ & N/A \\
\hline
\end{tabular}

Table 3: The QED LEC's from fits of $\delta m^{2}$. The pure QCD LEC's were taken from the SU(3) fit in [7]. The last column gives phenomenological results [2].

From Table 3 we can see that the LEC's change slightly from $16^{3}$ to $24^{3}$, but $C$ and $Y_{3}$ change significantly when the the log terms are included. The effect on the other LEC's is not so drastic. 
Also shown in Table 3 are the values from phenomenology [2], which are roughly consistent with ours within an order of magnitude, though we emphasize that our values do not include systematic corrections due to finite volume, explicit chiral symmetry breaking, and non-zero lattice spacing, to name just three, and so should be taken as preliminary.

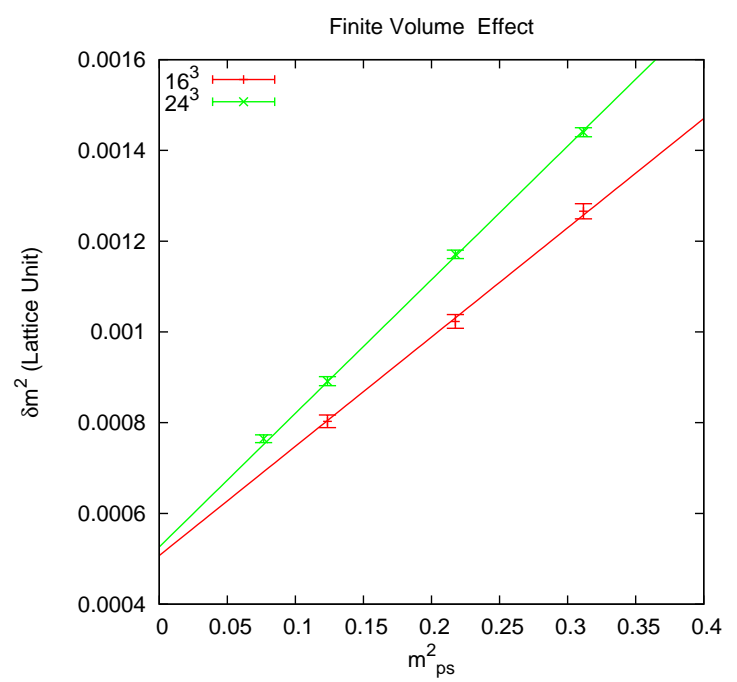

Figure 2: Finite volume effect on the $u \bar{d}$ meson.

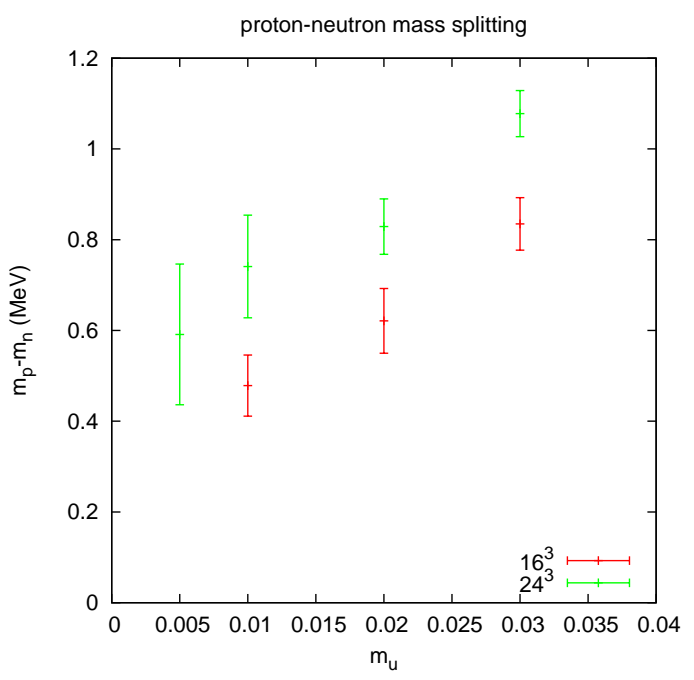

Figure 3: The proton-neutron mass difference.

Figure 2 shows the comparison of the charged meson splitting from $16^{3}$ and $24^{3}$ lattices, and the relative change is given in Table 4 . The difference actually decreases at the smallest quark mass, 0.01, which is why the finite volume effect shown in Figure 2 appears to go away in the chiral limit (linear fit). Interestingly, from Figure 1, one can see that the neutral splitting is mostly insensitive to the finite volume, at least for the volumes studied here. We are still investigating these effects, and plan a detailed comparison with finite volume chiral perturbation theory[9].

\begin{tabular}{|c|c|}
\hline$m_{\text {val }}$ & $2 \frac{\delta m^{2}\left(24^{3}\right)-\delta m^{2}\left(16^{3}\right)}{\delta m^{2}\left(24^{3}\right)+\delta m^{2}\left(16^{3}\right)}$ \\
\hline 0.01 & $0.1043(9)$ \\
\hline 0.02 & $0.1349(9)$ \\
\hline 0.03 & $0.1286(9)$ \\
\hline
\end{tabular}

Table 4: Finite volume effect on the $u \bar{d}$ meson.

Figure 3 is the result of the mass difference between the proton and neutron when the QED interaction is included. If there is no QED interaction and $m_{u}=m_{d}$, then $m_{n}=m_{p}$, which is the result of isospin symmetry. Figure 3 shows the mass difference for the unitary points. We can see the proton is heavier than the neutron and the mass difference decreases with quark mass. The $24^{3}$ result is larger than the $16^{3}$ result, once again signaling finite volume corrections. The baryon mass difference is noisier than in the pseudoscalar case. We plan to increase the statistics for the baryon mass spectrum to improve the precision on this result, and to compute the mass splitting coming from non-degenerate $u$ and $d$ quark masses, which is expected to switch the sign of the mass difference, in accord with Nature. 


\section{Summary and outlook}

Using 2+1 flavor QCD and non-compact QED, we have studied the electromagnetic mass splitting in hadrons. We have done our simulation on different sea quark mass ensembles and have used both $16^{3}$ and $24^{3}$ lattices to investigate the finite volume effect on the mass splitting.

Preliminary fits appear to be consistent with NLO PQ $\chi$ PT, including photons, for the parameters in our study, except at the heaviest quark mass, 0.03 , which is close to the physical strange quark mass. A more complete study of systematic errors, including finite volume and explicit chiral symmetry breaking effects, is in progress. Next we will use the LEC's obtained from our analysis to determine the physical, non-degenerate, $u, d$, and $s$ quark masses.

\section{Acknowlegements}

We thank the US Department of Energy and RIKEN for the support necessary to carry out this research. RZ and TB were supported by US DOE grant DE-FG02-92ER40716. Computations were carried out on the QCDOC supercomputers at the RIKEN BNL Research Center, BNL, and Columbia University.

\section{References}

[1] R. Dashen Chiral SU(3) $\otimes S U(3)$ as a symmetry of the strong interactions Phys. Rev. 183, (1969) 1245, arXiv:hep-lat/0708.0484.

[2] J. Bijnens and N. Danielsson Electromagnetic corrections in partially quenched chiral perturbation theory Phys. Rev. D. 75, (2007) 014505, arXiv:hep-lat/0610127.

[3] A. Duncan, E. Eichten and H. Thacker, Electromagnetic splittings and light quark masses in lattice QCD, Phys. Rev. Lett. 76, (1996) 3894, arXiv:hep-lat/9602005.

[4] S. Beane, K. Orginos and M. Savage Strong-isospin violation in the neutron proton mass difference from fully-dynamical lattice $Q C D$ and PQQCD Nucl. Phy. B. 768, (2007) 38-50, arXiv:hep-lat/0605014.

[5] D.J. Antonio et. al First results from $2+1$ - flavor domain wall QCD: Mass spectrum, topology change, and chiral symmetry with $L_{S}=8$ Phys. Rev. D. 75, (2007) 114501, arXiv:hep-lat/0612005.

[6] C. Allton et. al $2+1$ flavor domain wall QCD at $(2 \mathrm{fm})^{3}$ lattice: Light meson spectroscopy with $L_{S}=16$ Phys. Rev. D. 76, (2007) 014504, arXiv:hep-lat/0701013.

[7] C. Allton et. al Physical Results from 2+1 Flavor DomainWall QCD and SU(2) Chiral Perturbation Theory arXiv:hep-lat/0804.0703.

[8] T. Blum, T. Doi, M. Hayakawa, T. Izubuchi, and N. Yamada Determination of light quark masses from the electromagnetic splitting of pseudoscalar meson masses computed with two flavors of domain wall fermions Phys. Rev. D. 76, (2007) 114508, arXiv:hep-lat/0708.0484.

[9] M. Hayakawa and S. Uno, QED in finite volume and finite size scaling effect on electromagnetic properties of hadrons arXiv:hep-ph/0804.2044. 